\title{
Investigation of Correction Method of Recovery Effect and Motion Blur for SUV Quantification in FDG PET/CT in Patients with Early Lung Cancer
}

Noriyuki Kadoya $^{1 *}$, Yukio Fujita1, Kengo Ito', Suguru Dobashi², Ken Takeda², Kazuma Kishi ${ }^{3}$, Takaya Yamamoto', Rei Umezawa1, Toshiyuki Sugawara ${ }^{1}$, Tomohiro Kaneta ${ }^{4}$, Haruo Matsushita ${ }^{1}$ and Keiichi Jingu ${ }^{1}$

${ }^{1}$ Department of Radiation Oncology, Tohoku University School of Medicine, Sendai, Japan

${ }^{2}$ Department of Radiological Technology, School of Health Sciences, Faculty of Medicine, Tohoku University, Sendai, Japan

${ }^{3}$ Department of Radiation Technology, Tohoku University Hospital, Sendai, Japan

${ }^{4}$ Department of Diagnostic Radiology, Tohoku University School of Medicine, Sendai, Japan

\begin{abstract}
Objective: We investigated the effects of partial volume and respiratory motion using a National Electrical Manufacturers Association (NEMA) phantom and proposed a simple method for correction of maximum standardized uptake value $\left(\mathrm{SUV}_{\max }\right)$ for respiratory motion in early lung cancer.

Methods: The maximum recovery coefficient $(\mathrm{RC})$ in static mode were measured using the NEMA phantom and a dynamic moving platform. The phantom on the platform was either at rest or moving sinusoidally along the longitudinal axis of the scanner to simulate respiratory motion. We also calculated estimated RC using our approximation.

Results: RC of the sphere of $28 \mathrm{~mm}$ in diameter decreased from 0.96 to 0.80 and 0.41 with 20 and $50 \mathrm{~mm}$ of motion amplitude, respectively. For the sphere of $10 \mathrm{~mm}$ in diameter, RC was decreased from 0.40 to 0.18 and 0.08 with 20 and $50 \mathrm{~mm}$ of motion amplitude, respectively. Our results showed that RC decreased with increase in motion amplitude. Average percentage differences between measurement and estimation in the sphere of $37,28,22,17$, 13 and $10 \mathrm{~mm}$ were $-1.8 \pm 3.7,-3.1 \pm 11.3,-2.8 \pm 10.5,-8.1 \pm 6.6,-7.0 \pm 12.3$ and $-1.8 \pm 12.2 \%$, respectively. This result showed that our simple correction method could estimate SUV max $_{\text {mith }}$ moderate accuracy.

Conclusions: Our results clearly demonstrated that RC decreases with increase in motion amplitude, as expected. Our simple correction with moderate accuracy method could not precisely estimate RC. However, the estimated values agreed with the measurements. Thus, our methods could be used in clinical practice to calculate the approximate $\mathrm{SUV}_{\max }$ for lung cancer patients undergoing radiotherapy showing the malignancy grading for early lung cancer.
\end{abstract}

Keywords: SUV quantification; FDG PET/CT; Early lung cancer

\section{Introduction}

Whole body F-18 fluoro-2-deoxyglucose (18F-FDG) positron emission tomography/computed tomography (PET/CT) has become an important method for detecting tumors, planning radiation treatment and evaluating response to therapy. However, PET/CT imaging of the lung and abdomen region is generally affected by patient respiratory motion, which can lead to underestimation of maximum standardized uptake value $\left(\mathrm{SUV}_{\max }\right)$ of a region of interest, overestimation of tumor volume and mismatched PET and CT images that yield attenuation correction errors, registration errors and tumor mislocalization [1-3].

The effects of partial volume and respiratory motion on SUV were investigated in previous studies [1-9]. The most widely used method to correct for respiratory motion is repiratory-gated PET/CT, which divides PET data into different gates based on either temporal phase or respiratory displacement information with potential 4D CT for phase-matched attenuation correction. Guerra et al. showed that the respiratory-gated PET/CT technique is a valuable clinical tool for diagnosing lung lesions, improving quantification and confidence in reporting, reducing 3D undermined findings and increasing the overall accuracy in lung lesion detection and characterization [10]. However, there are few hospitals in Japan in which this technique has been introduced. It is better to correct SUV for respiratory motion in clinical practice. A study on 108 patients showed that $\mathrm{SUV}_{\max }$ increased as much as $51.8 \%$ on average from free-breathing PET to deepinspiration-breathhold PET/CT for lesions in the lower lung region [11]. However, the breath hold PET/CT cannot correct the partial volume effect. Though the usage is limited to lung cancer patients undergoing radiotherapy, we proposed a simple method for correction of SUV in the lung region using tumor size and motion displacement.

In this study, we investigated the effects of partial volume and respiratory motion using a phantom and the usefulness of a simple method to correct SUV for respiratory motion to know the actual malignancy in early lung cancer.

\section{Materials and Methods}

\section{Equipment and imaging protocol}

A PET-CT scanner (True Point Biograph 40, Siemens, Erlangen, Germany) was used to acquire data. The PET-CT scanner is composed of a lutetium oxyorthosilicate (LSO) crystal detector, 52 rings $(84.2 \mathrm{~cm}$ in diameter) and 32448 crystal numbers that give a transaxial field of view (FOV) of $60.5 \mathrm{~cm}$ and an axial FOV of $21.6 \mathrm{~cm}$.

*Corresponding author: Noriyuki Kadoya, Department of Radiation Oncology, Tohoku University School of Medicine, 1-1 Seiryo-chou, Aoba-ku, Senda 980-8574, Japan, Tel: +81-22-717-7312; Fax: +81-22-717-7316; E-mail: kadoya.n@rad.med.tohoku.ac.jp

Received June 25, 2013; Accepted August 26, 2013; Published August 29, 2013

Citation: Kadoya N, Fujita Y, Ito K, Dobashi S, Takeda K, et al. (2013) Investigation of Correction Method of Recovery Effect and Motion Blur for SUV Quantification in FDG PET/CT in Patients with Early Lung Cancer. J Nucl Med Radiat Ther 4: 162 doi:10.4172/2155-9619.1000162

Copyright: ( 2013 Kadoya N, et al. This is an open-access article distributed under the terms of the Creative Commons Attribution License, which permits unrestricted use, distribution, and reproduction in any medium, provided the original author and source are credited. 
Citation: Kadoya N, Fujita Y, Ito K, Dobashi S, Takeda K, et al. (2013) Investigation of Correction Method of Recovery Effect and Motion Blur for SUV Quantification in FDG PET/CT in Patients with Early Lung Cancer. J Nucl Med Radiat Ther 4: 162. doi:10.4172/2155-9619.1000162

Page 2 of 7

The imaging protocol used in this study is the most widely used method in clinical applications. CT data were acquired using a helical CT scan with scanning parameters of $140 \mathrm{kV}, 75 \mathrm{~mA}$, and $2.5 \mathrm{~mm}$ in slice thickness. Following the CT scan, PET data were acquired for 5 min per bed position. During the helical CT scan and PET emission scan, the targets in the phantom were either at rest or moving sinusoidally along the longitudinal axis of the scanner to simulate respiratory motion.

PET images $(168 \times 168 \times 47$ for a $60.5 \mathrm{~cm}$ transaxial FOV with a slice thickness of $2.5 \mathrm{~mm}$ ) were reconstructed using an ordered subset expectation maximization (OSEM) algorithm (14 subsets, 6 iterations and $8 \mathrm{~mm}$ FWHM Gaussian post-filter ) and were corrected for attenuation using the CT attenuation map in Hounsfield units (HUs). Corrections for scatter and random events were also incorporated in the OSEM algorithm. These reconstruction parameters are usually used in standard clinical protocols for patient scans.

\section{Phantom study}

The NEMA IEC body phantom shown in figure 1 was designed in accordance with the recommendations of the International Electrotechnical Commission (IEC) and modified by the National Electrical Manufacturers Association (NEMA) to evaluate whole-body PET performance. The phantom consists of a body phantom $(18 \mathrm{~cm}$ in length and $9.8 \mathrm{~L}$ ) and an inset with six hollow spheres of various sizes. In order to simulate respiratory motion using the NEMA phantom, we used a dynamic thorax platform (Model 008PL; Computerized Imaging Reference Systems, Norfolk, USA). The NEMA phantom was placed on the platform and moved along the longitudinal axis of the scanner to simulate respiratory motion.

\section{Recovery coefficients}

In order to investigate the effect of target size, phantoms with hollow spheres of $0.5,1.2,2.6,5.6,11.5$ and $26.5 \mathrm{ml}(10,13,17,22$, 28 and $37 \mathrm{~mm}$ in i.d., respectively) filled with ${ }^{18} \mathrm{~F}$-FDG were inserted. To simulate the measurement of ${ }^{18} \mathrm{~F}-\mathrm{FDG}$ for lung cancer, the NEMA phantom not filled with water was used. For data analysis, the recovery coefficient (RC) in the volume of interest (VOI) was defined as follows:

$$
\mathrm{RC}=\frac{A_{\text {observed }}}{A_{\text {static }, 37 \mathrm{~mm}}}
$$

Where $\mathrm{A}_{\text {observed }}$ is activity observed in the VOI (Bq) and $\mathrm{A}_{\text {static } 37 \mathrm{~mm}}$ is activity in the sphere of $37 \mathrm{~mm}$ in i.d. without movement. This value was regarded as 'true' activity in the VOI. $\mathrm{RC}_{\text {max }}$, calculated using the maximum activity concentration, is more sensitive to image noise than in $\mathrm{RC}_{\text {mean }}$, calculated using mean activity concentration in the VOI. However, $\mathrm{RC}_{\text {mean }}$ can easily vary based on the VOI, which is difficult to determine in a moving object [4]. Therefore, we used $\mathrm{RC}_{\text {max }}$ in this study because of its consistency in data analysis, especially for the respiratory induced moving target. To detect the image pixel having maximum activity concentration in each target sphere, MATLAB (The Math Works, Inc., Natick, MA) was used.

\section{Respiratory motion}

To simulate different respiratory speeds, the phantoms were translated sinusoidally with the following formula:

$$
\mathrm{y}(\mathrm{t})=y_{0}+A \sin ^{n}\left(\frac{\partial t}{\hat{o}}\right)
$$

where $\mathrm{y}_{0}$ is the baseline position at exhalation and $\mathrm{A}$ is the amplitude of motion. The peak-to-peak amplitudes were $0,5,10,20,30,40$ and $50 \mathrm{~mm}$. The respiratory cycle period, $\tau$ was fixed at $4.5 \mathrm{~s}$. This representative breathing period was determined from a previous paper [2].The value of $\mathrm{n}$ was set to 4 . A previous study showed that modeling the breathing curve using $n=4$ closely resembles free breathing motion since exhalation takes about twice as long as inhalation [12]. The curves are shown in figure 2.

\section{Simulation}

In order to investigate the effect of volume mismatch on attenuation correction, measurements with movement were simulated using

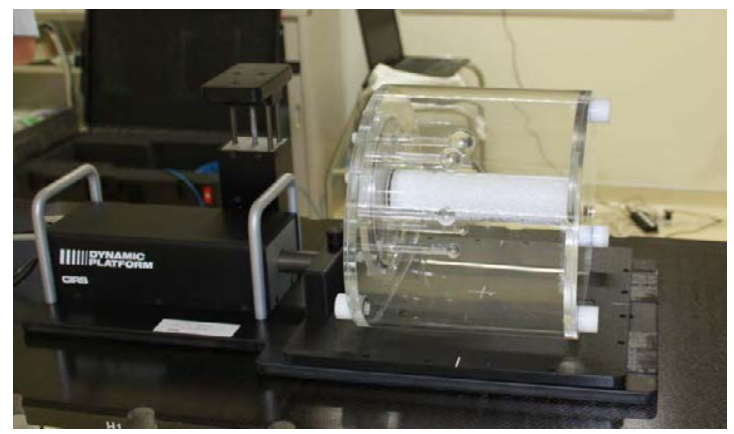

Figure 1: The NEMA phantom placed on the dynamic thorax platform. The phantom moved along the longitudinal axis of the scanner.

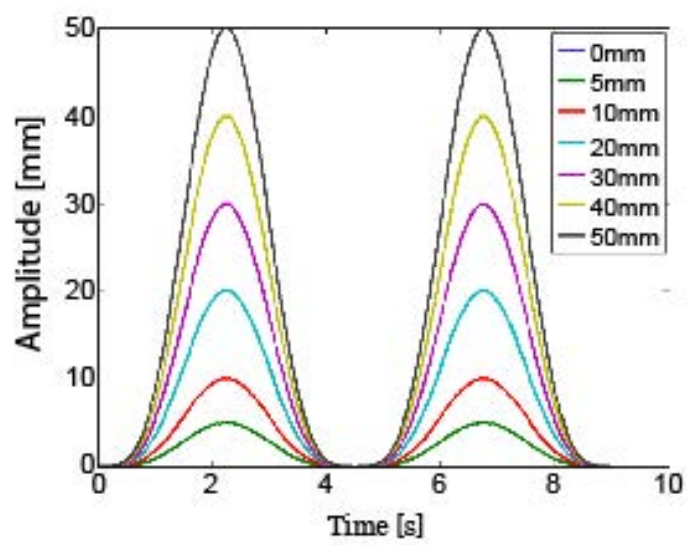

Figure 2: Motion curves of the dynamic thorax phantom. The peak-to-peak amplitudes were $0,5,10,20,30,40$ and $50 \mathrm{~mm}$.
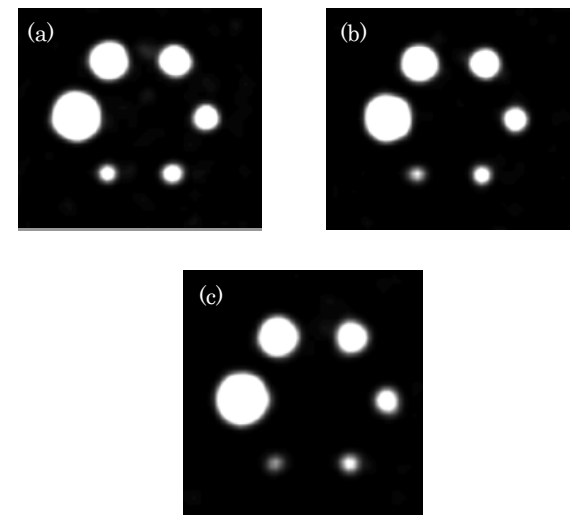

Figure 3: Axis images of PET with motion amplitudes of (a) 10, (b) 20 and (c) $40 \mathrm{~mm}$. 
Citation: Kadoya N, Fujita Y, Ito K, Dobashi S, Takeda K, et al. (2013) Investigation of Correction Method of Recovery Effect and Motion Blur for SUV Quantification in FDG PET/CT in Patients with Early Lung Cancer. J Nucl Med Radiat Ther 4: 162. doi:10.4172/2155-9619.1000162

measurement. First, we created the probability density function for the target location. Next, simulation of measurement with movement was calculated to convolute the static measurement with its probability density function.

\section{Results}

PET images are shown in figure 3. These images clearly show that the activity decreases with increased motion amplitude, as expected. Figure 4 shows $\mathrm{RC}$ as a function of sphere diameter. RC of the sphere of $28 \mathrm{~mm}$ in diameter decreased from 0.96 in a static mode to 0.80 and
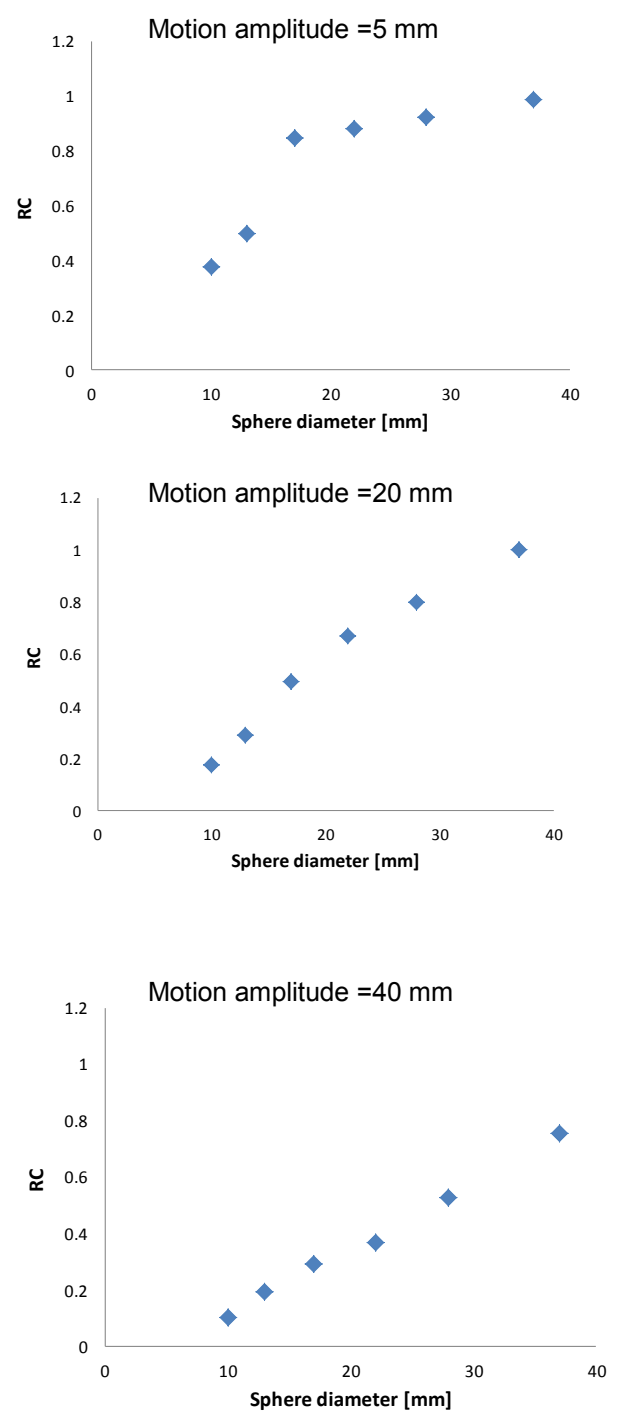

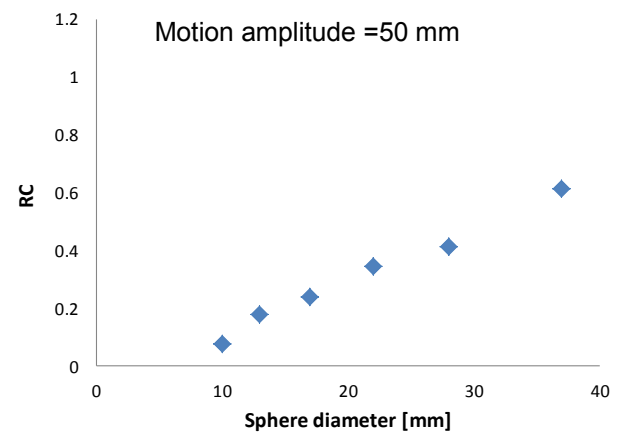

Figure 4: RC vs. sphere diameter. 
0.41 with 20 and $50 \mathrm{~mm}$ of motion amplitude, respectively. For the sphere of $10 \mathrm{~mm}$ in diameter, RC decreased from 0.40 to 0.18 and 0.08 with 20 and $50 \mathrm{~mm}$ of motion amplitude, respectively. Figure 5 shows RC as a function of motion amplitude. The results show that RC decreases with increase in motion amplitude.

Simulated RCs with sphere of 10,17 and $28 \mathrm{~mm}$ in diameter are shown in figure 6 . The results of simulation show almost the same trend of that of measurements. However, differences between results of simulations and measurements become large with increase in motion amplitude.

Next, we made a correction formula using obtained data. The formula was comprised of two terms: one term approximated the reduction by the partial volume effect and the other term approximated reduction by the motion blur. The first term was decided to fit the result with no motion using a sigmoid curve. Sigmoid curve made the goodness-of-fit measure between the estimated and measured values
(Figure 7). Second term was decided to fit the measurement using an exponential function. Finally, we made the following formulas to estimate the RC.

$\mathrm{RC}(\mathrm{x}, \mathrm{y})=\frac{a}{1+b \times \exp (-c x)} \times(d x+e) \exp \left(\frac{-y}{f}\right)($ Sphere diameter $>=13 \mathrm{~mm})$

$\mathrm{RC}(\mathrm{x}, \mathrm{y})=\frac{a}{1+b \times \exp (-c x)} \times \exp \left(\frac{-y}{d x+e}\right)($ Sphere diameter $<13 \mathrm{~mm})(4)$

Where $\mathrm{x}$ is sphere diameter, $\mathrm{y}$ is motion amplitude, and $\mathrm{a}, \mathrm{b}, \mathrm{c}, \mathrm{d}$, e and $\mathrm{f}$ are constant. When the estimated value in second term was greater than 1.0, we took 1.0 for the estimated value in second term. Estimated RC could be calculated by only two measured values (sphere diameter and motion amplitude). A comparison of measurements and estimations are shown in Figure 8. The estimated values using this approximation almost agreed with the measurements. Average percentage differences between measurement and estimation in the sphere of $37,28,22,17,13$
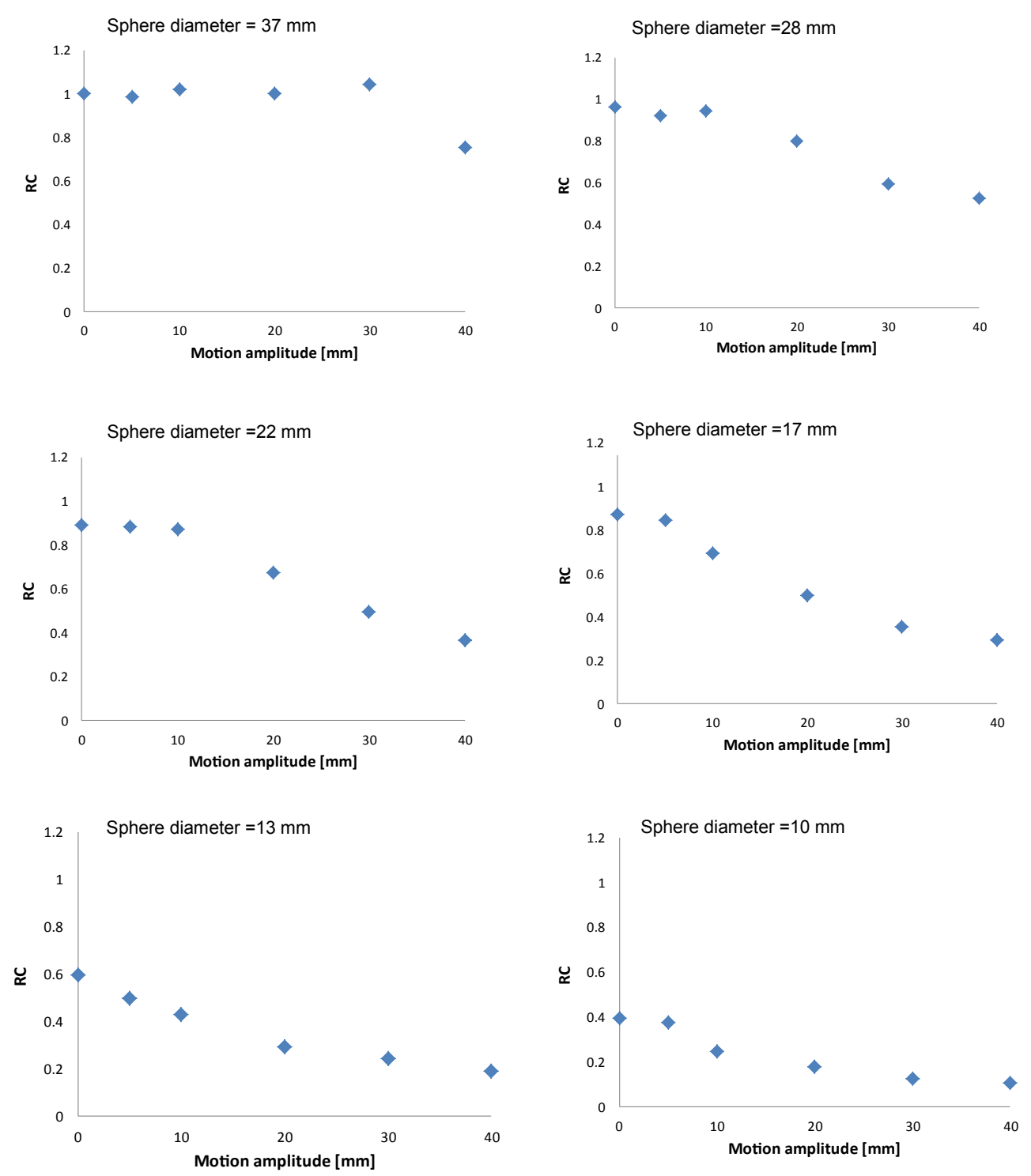

Figure 5: RC vs. motion amplitude. 


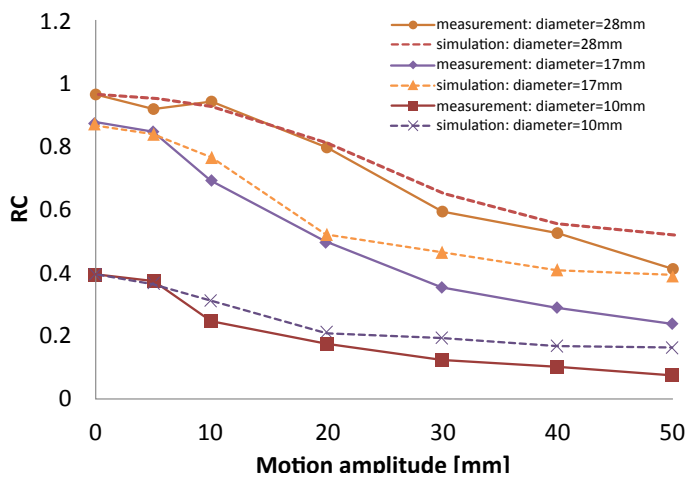

Figure 6: Comparison of measurements and simulated values.

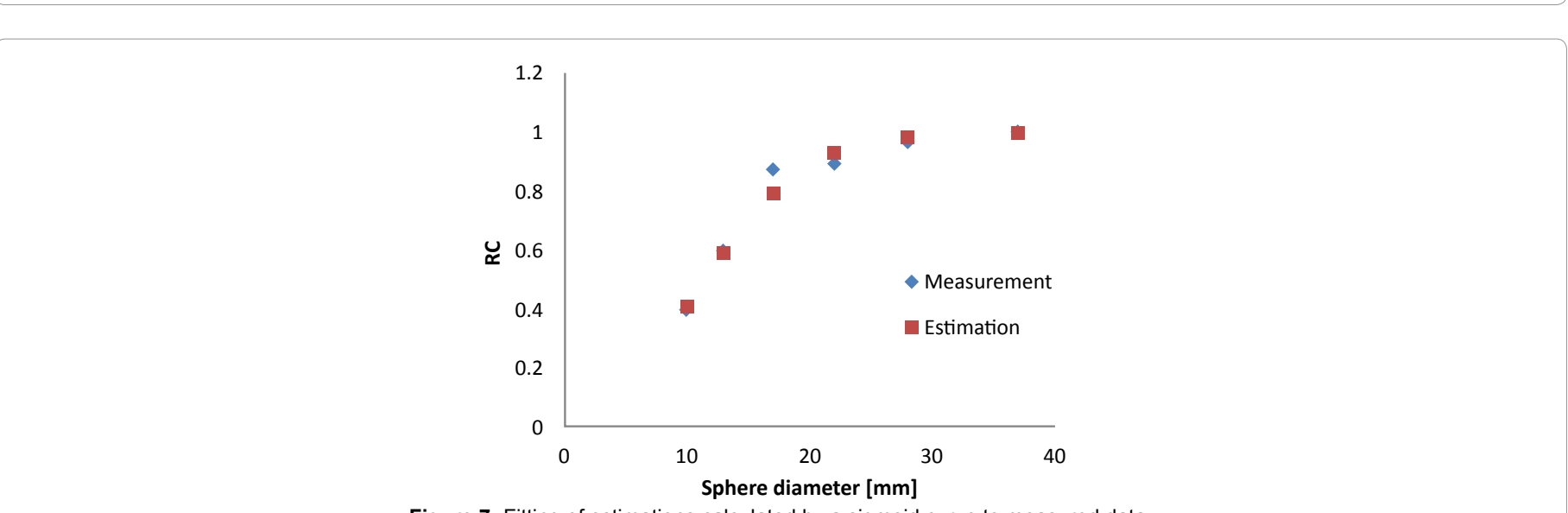

Figure 7: Fitting of estimations calculated by a sigmoid curve to measured data

and $10 \mathrm{~mm}$ were $-1.8 \pm 3.7,-3.1 \pm 11.3,-2.8 \pm 10.5,-8.1 \pm 6.6,-7.0 \pm 12.3$ and $-1.8 \pm 12.2 \%$, respectively. This result showed that the estimated values using this approximation had relatively good agreement with the measurements. Average percentage differences between measurement and estimation in the motion amplitude of $0,5,10,20,30,40$ and 50 mm were $-0.3 \pm 5.0,-1.0 \pm 7.1,2.2 \pm 6.2,2.0 \pm 4.6,-1.1 \pm 4.2,-9.5 \pm$ 6.9 and $-21.0 \pm 7.4 \%$, respectively. This result showed the differences between two values increased with increase in motion amplitude.

\section{Discussion}

In this study, we investigated the impact of respiratory motion and

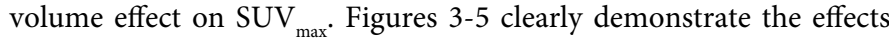
of these two factors and show that the measured value decreases with increase in motion amplitude, as expected. These findings corroborate those of Park et al. [2].

In the present study, the difference between measurements and simulated values became greater with increase in motion amplitude (Figure 6). In a PET/CT procedure, the difference in acquisition time of a CT scan (a few seconds) from that of a PET scan ( $5 \mathrm{~min}$ ) can lead to a misalignment between CT and PET images, resulting in an incorrect attenuation correction map and consequently incorrect $\mathrm{SUV}_{\text {max }}$ values. In addition, the image noise during a PET scan was not considered in the simulation since only the measurements in static mode were used. Liu et al. reported that $\mathrm{SUV}_{\max }$ measurement in clinical practice is affected by image noise [3]. These two factors may have a negative impact on the agreement of measured and simulated values. Our results showed that it was difficult to simulate RC with motion using the data with no motion.

For the correction method, the estimated value obtained by Equation (3) and (4) relatively had good agreement with the measurements. However, the differences increased with increasing the motion amplitude. We used an exponential function to fit the measurements. As mentioned above, measurement of $\mathrm{SUV}_{\max }$ was affected by the misalignment between attenuation correction $\mathrm{CT}$ and PET images. To estimate actual SUV ${ }_{\text {max }}$, the effect of this misalignment should in the equation. Furthermore, our purpose was not to estimate actual SUV but to estimate a reliable value using a simple method. Moreover, since the reproducibility of SUVmax measurements is poor, complete agreement of the estimated value with the measured value is meaningless [13].Thus, in clinical practice, correction of SUV using our equation is better than no correction. Since this correct method is simple and needs no additional device or no more imaging time and can use patient's data, it could be used in many hospitals in which respiratory motion artifacts are not corrected. However, when this approximation created by measurements in a phantom study is used for patients, it is necessary to consider the fact that the respiratory pattern and cycle is not regular in all patients. That is, as the estimated values were calculated using two values (tumor size and tumor displacement), there was no consideration of the change in respiratory pattern during acquisition of a PET image. Liu et al. analyzed a large number of respiratory traces (1295) and reported that about $40 \%$ were not regular respiratory cycles [3]. Furthermore, the actual tumor in the lung cancer moves in three dimensions. Further investigation of 

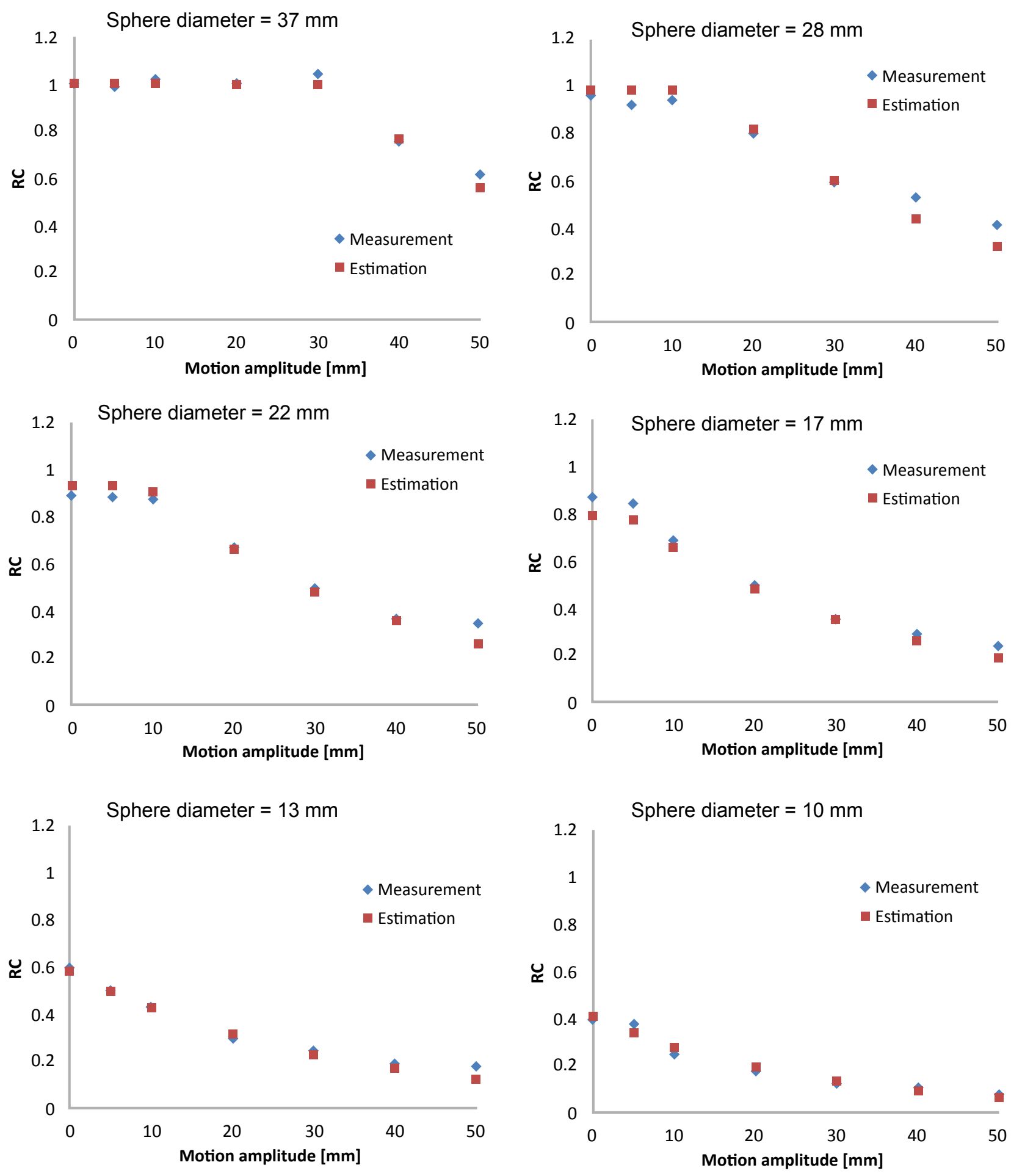

Figure 8: Comparison of measurements and estimation.

these effects of irregular respiratory and three-dimensional motion is necessary.

\section{Conclusions}

We investigated the impact of respiratory motion and volume effect

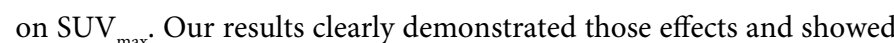
that the measured value decreases with increasing in motion amplitude and tumor diameter, as expected. Our simple correction method could estimate $\mathrm{SUV}_{\max }$ with moderate accuracy. Thus, our method could be used in clinical practice to calculate an approximate $\mathrm{SUV}_{\max }$ value showing actual degree of glucose metabolism in early lung cancer. 
Citation: Kadoya N, Fujita Y, Ito K, Dobashi S, Takeda K, et al. (2013) Investigation of Correction Method of Recovery Effect and Motion Blur for SUV Quantification in FDG PET/CT in Patients with Early Lung Cancer. J Nucl Med Radiat Ther 4: 162. doi:10.4172/2155-9619.1000162

\section{References}

1. Nehmeh SA, Erdi YE (2008) Respiratory motion in positron emission tomography/computed tomography: a review. Semin Nucl Med 38: 167-176.

2. Park SJ, Ionascu D, Killoran J, Mamede M, Gerbaudo VH, et al. (2008) Evaluation of the combined effects of target size, respiratory motion and background activity on 3D and 4D PET/CT images. Phys Med Biol 53: 3661 3679.

3. Liu C, Pierce LA 2nd, Alessio AM, Kinahan PE (2009) The impact of respiratory motion on tumor quantification and delineation in static PET/CT imaging. Phys Med Biol 54: 7345-7362.

4. Nehmeh SA, Erdi YE, Pan T, Pevsner A, Rosenzweig KE, et al. (2004) Fourdimensional (4D) PET/CT imaging of the thorax. Med Phys 31: 3179-3186.

5. Nehmeh SA, Erdi YE, Pan T, Yorke E, Mageras GS, et al. (2004) Quantitation of respiratory motion during 4D-PET/CT acquisition. Med Phys 31: 1333-1338.

6. Abdelnour AF, Nehmeh SA, Pan T, Humm JL, Vernon P, et al. (2007) Phase and amplitude binning for 4D-CT imaging. Phys Med Biol 52: 3515-3529.

7. Wolthaus JW, van Herk M, Muller SH, Belderbos JS, Lebesque JV, et al. (2005) Fusion of respiration-correlated PET and CT scans: correlated lung tumour motion in anatomical and functional scans. Phys Med Biol 50: 1569-1583.
8. Liu C, Alessio A, Pierce L, Thielemans K, Wollenweber S, et al. (2010) Quiescent period respiratory gating for PET/CT. Med Phys 37: 5037-5043.

9. Vines DC, Keller H, Hoisak JD, Breen SL (2007) Quantitative PET comparing gated with nongated acquisitions using a NEMA phantom with respiratorysimulated motion. J Nucl Med Technol 35: 246-251.

10. Guerra L, De Ponti E, Elisei F, Bettinardi V, Landoni C, et al. (2012) Respiratory gated $\mathrm{PET} / \mathrm{CT}$ in a European multicentre retrospective study: added diagnostic value in detection and characterization of lung lesions. Eur J Nucl Med Mol Imaging 39: 1381-1390.

11. Kawano T, Ohtake E, Inoue T (2008) Deep-inspiration breath-hold PET/CT of lung cancer: maximum standardized uptake value analysis of 108 patients. $J$ Nucl Med 49: 1223-1231

12. Ionascu D, Jiang SB, Nishioka S, Shirato H, Berbeco RI (2007) Internalexternal correlation investigations of respiratory induced motion of lung tumors. Med Phys 34: 3893-3903.

13. de Langen AJ, Vincent $A$, Velasquez LM, van Tinteren $H$, Boellaard $R$, et al. (2012) Repeatability of 18F-FDG uptake measurements in tumors: a metaanalysis. J Nucl Med 53: 701-708. 